\title{
Generation of inner ear hair cells in vitro from mouse embryonic stem cells
}

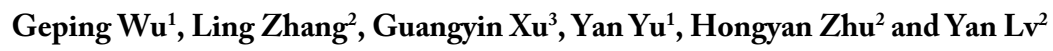 \\ ${ }^{1}$ Department of Otolaryngology, First People Hospital of Zhangjiagang City, Suzhou, 215600, China \\ ${ }^{2}$ Center of Translational Medicine, First People Hospital of Zhangjiagang City, Suzhou, 215600, China \\ ${ }^{3}$ Institute of Neuroscience and Department of Neurobiology and Psychology, Key lab of Pain Research and Therapy, Soochow University, Suzhou, 215123, China
}

\begin{abstract}
Embryonic stem cells (ESCs) are key tools for genetic engineering and development of stem cell-based therapies. The investigation of ESCs pluripotency and early lineage commitment is an essential precondition for its translational use. In this study, we explored the ability of mouse ESC to differentiate into inner ear hair cell lineages. Mouse embryonic stem cells (E14TG2A) were cultured on primary embryonic mouse fibroblasts (PMEF), and induced by successive steps with EGF and IGF-1 for 10 days to establish the formation of embryoid bodies (EBs), and then treated with bFGF for 8 days to induce potential inner ear hair cells. Quantitative real-time PCR and immunofluorescence analysis were performed, which demonstrated that presence and significant increased expression of the specific biomarkers for inner ear hair cells, Espin, myosin VIIa, Math1, a9 AchR proteins in the induced lineages. Our study provided the evidences that mESCs can be driven to express key genes in the development of inner ear hair cells and thereby promotes their status as candidate for regenerative therapies.
\end{abstract}

\section{Introduction}

Hearing loss is one of the most common disabilities all around the world. Approximately $12 \%$ to $17 \%$ of American adults (roughly 40 million people) show some degree of hearing loss [1]. Moreover, a large number of children in the United States are diagnosed with conductive hearing loss due to Eustachian tube dysfunction, or malformation of middle and external ear, between 2 and 3 out of every 1,000 children have some degree of congenital sensorineural hearing loss (SNHL) [2]. The prevalence of hearing disorders combined with the lack of recovery makes the treatment of congenital SNHL as a major challenge in the area of otology and audiology.

In most cases of SNHL, the primary pathology is the loss of mechanosensory hair cells located within the sensory epithelium of the cochlea [2]. Hair cells in the inner ear are essential for hearing and balance [3]. These hair-like structures are stereocilia, and they bend in response to sound. There are two types-auditory and vestibular [3]. Auditory hair cells are located in the cochlea of the inner ear, and are responsible for detecting sounds. Movements of the hairs are translated into electrical signals which are transmitted to the brain and interpreted as sound. There are also hair cells responsible for our sense of balance [3]. These are located in a different part of the inner ear - the vestibular organs - and are called vestibular hair cells. While damaged auditory hair cells can be compensated for with the use of hearing aids, there is no replacement or cure for damaged vestibular hair cells. Hair cells do not function in isolation, but need to be connected to the auditory centers of the brain through nerve fibers. The challenge of hair cell replacement is not only to replace damaged hair cells. New research, however, is showing that there may be a way to regenerate damaged hair cells.

Current approaches were proposed toward hair cell regeneration in the inner ear [4-6]. Understanding of the developmental biology of sensory cells in the inner ear should provide molecular pathways for the generation of hair cells from stem/progenitor cells [4-6]. Gene therapies and drug delivery systems designed for endogenous repair of inner ear sensory epithelia will affect the number of progenitor cells and their cell fate. The in vitro generation of inner ear hair cells from embryonic stem (ES) cells is a promising approach to produce cells suitable for deafness repair and cell-based replacement therapies of the auditory system.

\section{Materials and methods}

\section{Animal experiment protocol}

Animal experiments were approved by the Animal Care Committee of First People Hospital of Zhangjiagang City, and were conducted according to institutional guidelines for animal care.

\section{Primary embryonic mouse fibroblasts (PMEF)}

Freshly dissected mouse embryos of pathogen-free ICR mouse (Day 11-13) were minced and then incubated with Pierce MEF Isolation Enzyme (with papain) (\#88290) for 25 to 30 minutes and washed twice with Hanks Balanced Salt Solution (HBSS). For do-it-yourself trypsinbased isolation (DIY Trypsin), mouse embryonic tissue was incubated with $0.25 \%$ trypsin for 25 to 30 minutes and washed twice HBSS. Remaining steps of the both procedures were the same. The tissue was disrupted in Complete DMEM for Primary Cell Isolation (\#88287)

Correspondence to: Geping $\mathrm{Wu}, \mathrm{MD}, \mathrm{PhD}$. Department of Otolaryngology, First People Hospital of Zhangjiagang City, Suzhou, 215600, China. Tel: 86512 56919813; Fax: 86512 56919800,E-mail: gordon-wu@qq.com

Key words: mouse embryonic stem cells, inner ear hair cells, in vitro

Received: April 20, 2015; Accepted: May 05, 2015; Published: May 08, 2015 
by pipetting up and down 15 to 20 times with a pipette fitted with a $1000 \mu \mathrm{L}$ tip to generate a single cell suspension. Total cell yield was determined using an Invitrogen ${ }^{\text {ma }}$ Countess $^{\text {rnt }}$ Automated Cell Counter and cell viability was determined by trypan blue exclusion assay.

\section{Inner ear hair cells induction}

Mouse embryonic stem cells (E14TG2A) were from Cell Bank, Stem Cell Bank, Shanghai Institutes for Biological Sciences, Chinese Acedemy of Sciences. To induce differentiation of mES cells into inner ear hair cells, mES cells need to be separated from PMEF and cultivated in a suspension environment. Gelatin coated flasks are used to separate PMEF from mES cells. When mES colonies are ready for inner ear hair cells induction (defined as differentiation day 0), coat 2 T150 flasks with $0.1 \%$ gelatin $(18 \mathrm{ml} /$ flask) for $30 \mathrm{~min}$ at room temperature and then remove excess gelatin and wash with $1 \mathrm{x}$ PBS three times. Carefully remove media by aspiration, making sure not to dislodge loosely attached colonies. Add $10 \mathrm{ml}$ PBS briefly and then remove by aspiration to complete wash step. To separate mES cells from PMEF, add $0.25 \%$ trypsin/EDTA ( $3 \mathrm{ml} / \mathrm{dish}$, StemCell Technologies) and incubate for $3-5 \mathrm{~min}$ at $37^{\circ} \mathrm{C}$ until the stem cells and PMEF detach from the plate. Add $15 \mathrm{ml}$ fresh ES media without LIF and pipet cells up and down to break colonies (about 15-20 times). Then, add to a $0.1 \%$ gelatin-coated T150 flask and incubate for $30 \mathrm{~min}$ at $37^{\circ} \mathrm{C}$. After incubation, PMEF should attach to the gelatinized surface while ES cells should be floating. Collect the floating mES cells into a $50 \mathrm{ml}$ tube. Spin down at $800 \mathrm{rpm}$ and resuspend cells in $10 \mathrm{ml}$ of fresh Differentiation Medium (20 ng/ml EGF, $50 \mathrm{ng} / \mathrm{ml} \mathrm{IGF-1} \mathrm{for} 10$ days). Count the cells using a hemocytometer and then plate approximately $2 \times 10^{6}$ $\mathrm{mES}$ cells onto a $100-\mathrm{mm}$ bacterial or suspension culture dish. These plates permit growth of suspension cultures and promote formation of embryoid bodies (EBs). On differentiation day 1, mES cells form small floating aggregates (EBs) that are visible under a light microscope. Any carryover PMEF attach to the dish. Transfer the suspension cells and media into a $15 \mathrm{ml}$ tube and centrifuge at low speed (500 rpm) for $3 \mathrm{~min}$. Aspirate media carefully, add $10 \mathrm{ml}$ of fresh Differentiation Medium (10 $\mathrm{ng} / \mathrm{ml} \mathrm{bFGF}$ for 8 days) to the pelleted EBs, and then plate cells in a new bacterial dish. In addition to replenishing the media, this step removes any PMEF that carry over from the prior step.

\section{Quantitative Real-time PCR}

To determine the expression of mouse Espin, myosin VIIa, Math1, and $\alpha 9$ AchR, cDNA in mESC and induced mESC populations was synthesized using SuperScript II reverse transcriptase with random hexamers (Life Technologies). Melting curve analysis was done at the end of the reaction to assess the quality of the final PCR products. The threshold cycle $\mathrm{C}(\mathrm{t})$ values were calculated by fixing the basal fluorescence at 0.05 units. Three replicates were used for each sample, and the average $\mathrm{C}(\mathrm{t})$ value was calculated. The $\Delta \mathrm{C}(\mathrm{t})$ values were calculated as C(t) sample - C(t) Gapdh. The $\mathrm{N}$-fold increase or decrease in expression was calculated by the $\Delta \Delta \mathrm{Ct}$ method using the $\mathrm{C}(\mathrm{t}) \mathrm{Gapdh}$ value as the reference point.

\section{Immunofluorescence}

Immunofluorescence was performed as described previously [7]. Mouse stimulated ESCs growing on 20\% FBS-pre-coated cover-slides were fixed in $4 \%$ formaldehyde for $20 \mathrm{~min}$. After permeabilization with $0.5 \%$ Triton X-100 for 2 min, cells were blocked in PBS containing $5 \%$ BSA for $1 \mathrm{~h}$. Cells were then incubated with a mixture of rabbit antibodies against Espin, myosin VIIa, Math1, $\alpha 9$ AchR overnight at $4^{\circ} \mathrm{C}$. Cells were washed 3 times and then incubated with Goat anti- rabbit IgG H\&L (FITC) for 1 hour and mounted with DAPI-containing mounting solution. Fluorescent images were taken with a Zeiss confocal microscope (Carl Zeiss Microsystems).

\section{Statistical analysis}

All analyses were performed using GraphPad PRISM 4.0 (San Diego, CA). Results are expressed as the mean \pm SEM and were compared by Student $t$ test or analysis of variance as appropriate. Differences were considered significant at $\mathrm{P}$ less than or equal to 0.05 .

\section{Results}

\section{Embryoid bodies from mESC were generated in presence of the factors}

Embryoid bodies, generated from ESCs and iPSCs, were treated with the Wnt inhibitor $\operatorname{Dkk1}(8)$, the selective inhibitor of Smad3 (SIS3) that interferes with TGF-b signaling [9], and IGF-1, either alone or in combinations. To induce differentiation of mES cells into inner ear hair cells, $\mathrm{mES}$ colonies are ready for inner ear hair cells induction (defined as differentiation day 0 ) with $0.1 \%$ gelatin for $30 \mathrm{~min}$ at room temperature and then remove excess gelatin and wash with 1x PBS three times. On differentiation day $1, \mathrm{mES}$ cells formed small floating aggregates (EBs) using Differentiation Medium (20 ng/ml EGF, 50 ng/ ml IGF-1 for 10 days). Any carryover PMEF attach to the dish. Transfer the suspension cells and media into a $15 \mathrm{ml}$ tube and centrifuge at low speed $(500 \mathrm{rpm}$ ) for $3 \mathrm{~min}$. Aspirate media carefully, add $10 \mathrm{ml}$ of fresh Differentiation Medium (10 $\mathrm{ng} / \mathrm{ml}$ bFGF for 8 days) to the pelleted the formation of embryoid bodies (EBs), and then plate cells in a new bacterial dish. Embryoid body-derived cells were formed and attached to culture dishes (Figure 1). Formation of EBs could be more precisely controlled by the inoculation of known cell densities within single drops (10-20 $\mu \mathrm{L}$ ) suspended from the lid of a Petri dish, known as hanging drops. Formation of EBs in suspension is amenable to the formation of large quantities of EBs, but provides little control over the size of the resulting aggregates, often leading to large, irregularly shaped EBs. These EBs recapitulate many aspects of cell differentiation during early embryogenesis, and play an important role in the differentiation of ES cells into a variety of cell types in vitro.

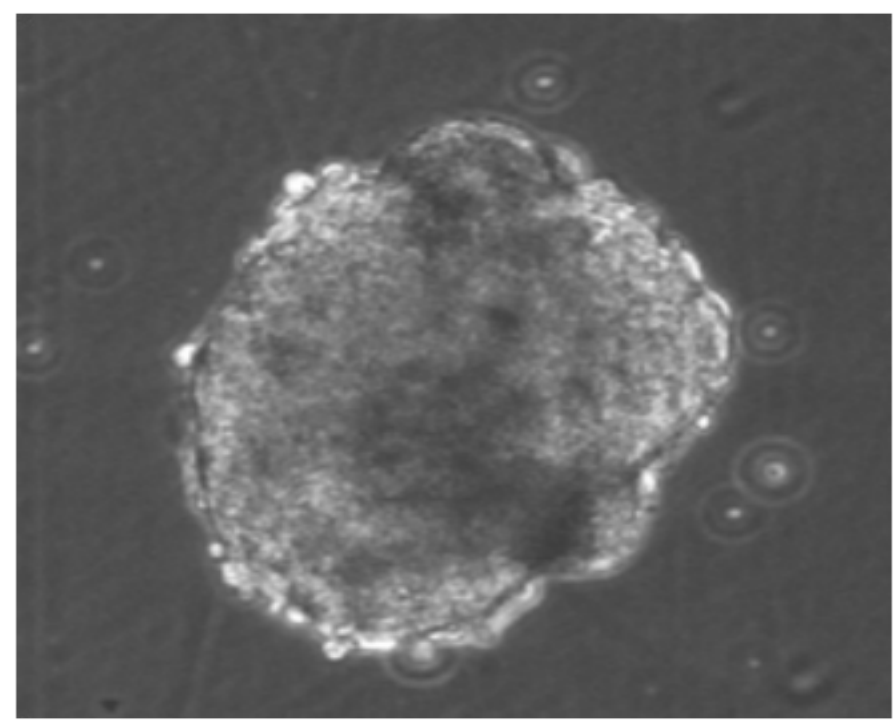

Figure 1. Embryoid bodies from $\mathrm{mESC}$ were generated in presence of the factors. 


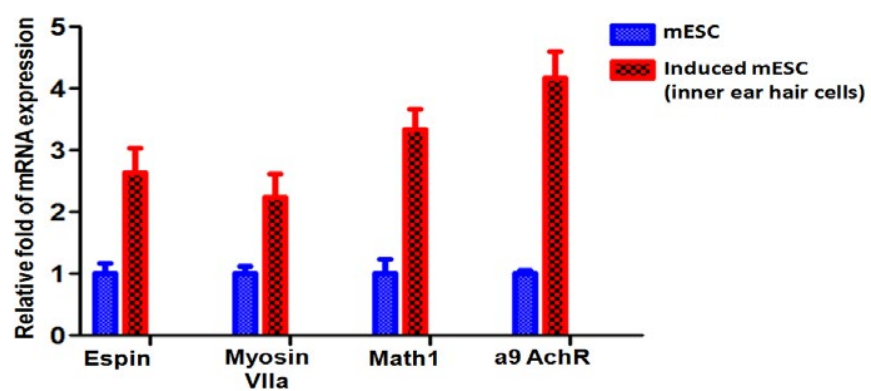

Figure 2. Quantitative real-time PCR analyses for expression of Nanog and the early otic markers Espin, myosin VIIa, Math1, $\alpha 9$ AchR. Data shown is repeated with three independent experiments and shown as mean \pm SEM. Gapdh were used as controls to normalize data.
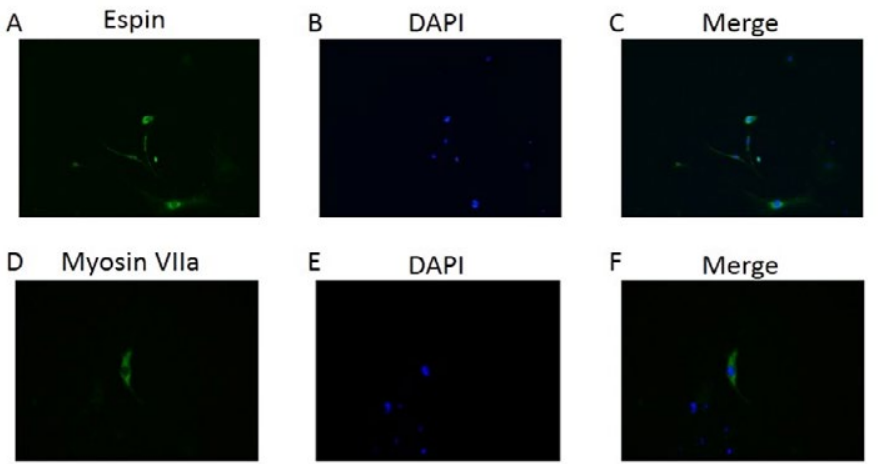

G
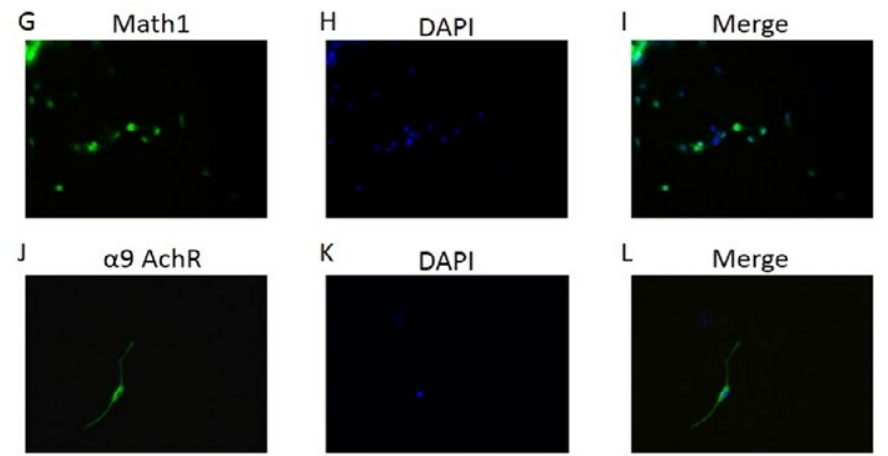

K
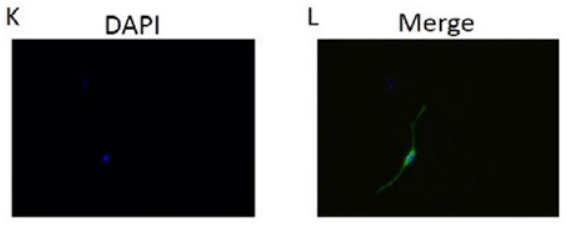

Figure 3. Characterization of mES cell-derived inner ear hair cells. Immunofluorescence staining for Espin, myosin VIIa, Math1, $\alpha 9$ AchR for FITC staining (Green). DAPI (blue) was used to identify the nuclei. X400. Panels A, D, G, J showed inner ear differentiation biomarkers expression; B, E, H, K demonstrated DAPI stained cell nuclear. C, F, I, L demonstrated the merge of staining.

\section{Characterization of mES cell-derived inner ear hair cells}

Within the context of ESC differentiation protocols, EB formation is often used as a method for initiating spontaneous differentiation toward the three germ lineages. EB differentiation begins with the specification of the exterior cells toward the primitive endoderm phenotype [10]. We next characterized the phenotype of the induction using the specific biomarkers for inner ear hair cells. Quantitative real-time PCR were performed on the induced inner ear hair cells population, which demonstrated that messenger RNA expression of Nanog and the early otic markers Espin, myosin VIIa, Math1, a9 AchR were significantly increasingly expressed in induced population when compared to those in mESC cells (Figure 2). Furthermore, immunofluorescence analysis also demonstrated that presence and significant increased expression of Espin, myosin VIIa, Math1, a9 AchR protein in the induced inner ear hair cells population (Figure 3). These data indicated successful generation of inner ear hair cells in vitro from mouse embryonic stem cells.

\section{Discussion}

In the present study, we presented the evidence that mouse ESC can be successfully differentiated into inner ear hair cells lineages. The quality of mES cells is the most critical parameter for efficient generation of inner ear hair cells. mES cells must be cultivated on PMEF to prevent spontaneous differentiation. Addition of LIF helps maintain the mES cells in an undifferentiated state. As mES cells divide every 12-15 h, the culture medium becomes depleted rapidly and must be replaced daily. To further enhance differentiation efficiency, we add a 10-day hair cells induction step prior to the inner ear hair cells specification step. This approach takes advantage of the fact that both IGF-1 and FGF signaling are crucial for the early stage of hair cell induction when embryonic cells first enter the inner ear cell lineage. In addition to hair cell induction, FGF signaling maintains cultures as inner ear hair cells. Incubation of bFGF in the developing inner ear hair cells leads to a dramatic expansion of inner ear hair cells population. A combination of FGFs and IGF-1 acts synergistically in inducing hair cells formation by promoting a posterior inner ear hair cells identity. Thus the 10-day hair cell population induction step is designed to direct the mES cells towards the hair cells lineage prior to initiation of the process of inner ear hair cells specification.

It is known that the morphological events and cell fate decisions that occur during mammalian inner ear development [4-6]. Development of the membranous labyrinth at the corresponding embryonic stage is shown in the middle column. At E9, common inner ear stem cells form an ectodermal thickening, the otic placode, which is located adjacent to rhombomeres 5 and 6 of the neural plate. The cross section of the neural plate and the otic placode demonstrated in the middle column. By E10.5, the otic placode invaginates and closes to form an epithelial sac, the otocyst. Next, the otocyst undergoes morphogenetic changes, including the extension of the endolymphatic duct and sac in the posterior dorsal and the elongation of the cochlear duct in the anterior ventral. Finally, by this stage the rudiment of the statoacoustic ganglia has formed adjacent to the otocyst. At E13.5, the cochlear duct is less than a single turn and cells have reached the prosensory stage. By E17, the cochlear duct has grown nearly its complete length and sensory cell commitment is largely complete. As discussed, the ability to isolate, expand, and then direct stem cells toward a specific cell fate holds great potential for both clinical and basic auditory research. However, a key step in this process is the demonstration that an isolated stem cell can develop as a functional hair cell. Oshima et al. [11] recently demonstrated that a stepwise protocol that mimics early embryonic development can be used to direct ESCs toward an inner ear, and, subsequently, a hair cell fate. ESC-induced hair cells generated in this manner express genes that are consistent with hair cell formation and develop a stereocilliary bundle-like protrusion that is consistent with a hair cell phenotype. Most importantly, electrophysiological studies demonstrate functional stereociliary bundles and mechanosensitivity in these ESC-derived hair cells. While these results offer definitive proof of the ability to drive ESCs to develop as functional hair cells in vitro, it is important to consider several caveats.

At the otic placode stage, all of the cells within the placode express the transcription factors Pax8, Dlx5, and Pax2 [12]. Pax8 is the first of these genes to be expressed, suggesting a possible role in otic 
induction. However, inner ear development is normal in Pax8 null mice [13] indicating that the effects of Pax8 are either redundant with another gene or may be compensated for in response to deletion. Dlx5 becomes restricted to the dorsolateral compartment of otic vesicle and deletion affects the morphogenesis of sensory and nonsensory vestibular structures, characterized by the absence of one to three of the semicircular canals and a shortening of the endolymphatic duct [14]. Deleting Pax2 in the mouse inner ear leads to agenesis or severe malformation of the cochlea along with varying degrees of defects in the vestibular region of the inner ear and in the auditory and vestibular ganglia [15]. A number of studies have demonstrated that the bHLH protein Atoh1 (formerly known as Math1) is a commitment factor that drives prosensory cells to develop as hair cells. All hair cells, both auditory and vestibular, are absent in Atoh1 mutant mice [16] and forced expression of Atoh1 is sufficient to induce a hair cell fate both within the prosensory domain [17] and even in nonsensory regions of the cochlea. Nonsensory cells in the mature cochlea retain the competence to generate new hair cells after overexpression of Math1 in vivo and that Math1 is necessary and sufficient to direct hair cell differentiation in these mature nonsensory cells [18]. Espin contains an additional actin-binding site in its $\mathrm{N}$ terminus and is a major actinbundling protein of the Sertoli cell-spermatid ectoplasmic specialization junctional plaque [19]. Mutation of deaf jerker mouse demonstrated that the gene encoding the espin actin-bundling proteins of hair cell stereocilia and lacks espins [20]. In addition, structural polymorphism of the actin-espin system demonstrated that a prototypical system of filaments and linkers in stereocilia [21].

Activation of an endogenous stem cell population within the inner ear would probably represent the ideal source for repair of the sensory epithelia. However, existing data suggest that the number of endogenous stem cells in a mature cochlea may be low to nonexistent. Moreover, the procedures/treatments needed to activate these cells remain unknown. Therefore, an alternative strategy might be to transplant exogenous stem cells into the inner ear. This approach would offer several advantages, such as the ability to activate and bias stem cells toward an inner ear or hair cell fate prior to transplantation, as well as several challenges, with the foremost being the difficulty in targeting transplanted cells to the correct portion of the cochlea. Delivering cells directly to the organ of Corti would be ideal but poses several challenges related to its small size and relative inaccessibility. Transplantation of stem cells to the spiral ganglion appears to be a more promising approach. Increasing the number of neurons within the ganglion could benefit the efficacy of cochlear implant. Stria vascularis and spiral ligament. A substantial percentage of congenital hearing loss is caused by mutations in genes related to ion transport, gap junctional communication, and endolymphatic homeostasis. Therefore, transplantation of stem cells that express wildtype alleles of some of these genes could be an option for the treatment of hearing loss. A final strategy might be to transplant genetically engineered cells that produce and secrete factors that might protect hair cells or attract spiral ganglion peripheral axons into the scala vestibuli or scala media.

In conclusion, our study demonstrated that the potential ability of mouse ESC to differentiate into inner ear hair cell lineages. Further study will be performed on transplanting these "potential hair cells" into animal model with hearing loss, which may be considered as a future potential treatment of human deafness.

\section{Disclosures}

The authors of the manuscript have no financial conflict of interest to disclose.

\section{Acknowledgements}

This study was supported by ZhangJiaGang Science-technology Supporting Plan (ZKS1318, 2013).

\section{References}

1. Lam BL, Lee DJ, Gomez-Marin O, Zheng DD, Caban AJ (2006) Concurrent visual and hearing impairment and risk of mortality: the National Health Interview Survey. Arch Ophthalmol 124: 95-101. [Crossref]

2. Mehra S, Eavey RD, Keamy DG Jr (2009) The epidemiology of hearing impairment in the United States: newborns, children, and adolescents. Otolaryngol Head Neck Surg 140: 461-72. [Crossref]

3. Devarajan K, Staecker H, Detamore MS (2011) A review of gene delivery and stem cell based therapies for regenerating inner ear hair cells. J Funct Biomater 2: 249-70. [Crossref]

4. Kamiya K (2015) Inner ear cell therapy targeting hereditary deafness by activation of stem cell homing factors. Front Pharmacol 6: 2. [Crossref]

5. Limb CJ (2012) When inner ear stem cell therapy becomes a reality. Trends Amplif 16: 3. [Crossref]

6. Okano T, Kelley MW (2012) Stem cell therapy for the inner ear: recent advances and future directions. Trends Amplif 16: 4-18. [Crossref]

7. Liu G, Park YJ, Abraham E (2008) Interleukin-1 receptor-associated kinase (IRAK) -1-mediated NF-kappaB activation requires cytosolic and nuclear activity. Faseb $J 22$ : 2285-96. [Crossref]

8. Glinka A, Wu W, Delius H, Monaghan AP, Blumenstock C, Niehrs C (1998) Dickkopf-1 is a member of a new family of secreted proteins and functions in head induction. Nature 391: 357-62. [Crossref]

9. Jinnin M, Ihn H, Tamaki K (2006) Characterization of SIS3, a novel specific inhibitor of Smad3, and its effect on transforming growth factor-beta1-induced extracellular matrix expression. Mol Pharmacol 69: 597-607. [Crossref]

10. Chen Y, Li X, Eswarakumar VP, Seger R, Lonai P (2000) Fibroblast growth factor (FGF) signaling through PI 3-kinase and Akt/PKB is required for embryoid body differentiation. Oncogene 19: 3750-6. [Crossref]

11. Oshima K, Shin K, Diensthuber M, Peng AW, Ricci AJ, et al. (2010) Mechanosensitive hair cell-like cells from embryonic and induced pluripotent stem cells. Cell 141: 70416. [Crossref]

12. Noramly S, Grainger RM (2002) Determination of the embryonic inner ear. J Neurobiol 53: 100-28. [Crossref]

13. Mansouri A, Chowdhury K, Gruss P (1998) Follicular cells of the thyroid gland require Pax8 gene function. Nat Genet 19: 87-90. [Crossref]

14. Acampora D, Merlo GR, Paleari L et al. (1999) Craniofacial, vestibular and bone defects in mice lacking the Distal-less-related gene Dlx5. Development 126: 3795-809. [Crossref]

15. Burton Q, Cole LK, Mulheisen M, Chang W, Wu DK (2004) The role of Pax2 in mouse inner ear development. Dev Biol 272: 161-75. [Crossref]

16. Bermingham NA, Hassan BA, Price SD, Vollrath MA, Ben-Arie N, et al. (1999) Math1: an essential gene for the generation of inner ear hair cells. Science 284: 183741. [Crossref]

17. Jones JM, Montcouquiol M, Dabdoub A, Woods C, Kelley MW (2006) Inhibitors of differentiation and DNA binding (Ids) regulate Math1 and hair cell formation during the development of the organ of Corti. J Neurosci 26: 550-8. [Crossref]

18. Kawamoto K, Ishimoto S, Minoda R, Brough DE, Raphael Y (2003) Math1 gene transfer generates new cochlear hair cells in mature guinea pigs in vivo. J Neurosci 23 : 4395-400. [Crossref]

19. Chen B, Li A, Wang D, Wang M, Zheng L, et al. (1999) Espin contains an additiona actin-binding site in its $\mathrm{N}$ terminus and is a major actin-bundling protein of the Sertoli cell-spermatid ectoplasmic specialization junctional plaque. Mol Biol Cell 10: 4327-39. [Crossref] 
Geping Wu (2015) Generation of inner ear hair cells in vitro from mouse embryonic stem cells

20. Zheng L, Sekerkova G, Vranich K, Tilney LG, Mugnaini E, et al. (2000) The deaf jerker mouse has a mutation in the gene encoding the espin actin-bundling proteins of hair cell stereocilia and lacks espins. Cell 102: 377-85. [Crossref]
21. Purdy KR, Bartles JR, Wong GC (2007) Structural polymorphism of the actin-espin system: a prototypical system of filaments and linkers in stereocilia. Phys Rev Lett 98: 058105. [Crossref]

Copyright: (C2015 Geping Wu. This is an open-access article distributed under the terms of the Creative Commons Attribution License, which permits unrestricted use, distribution, and reproduction in any medium, provided the original author and source are credited. 